\title{
A CONSTRUÇ̃̃O E O USO DE SISTEMAS DE CATEGORIAS PARA AVALIAR O ENTENDIMENTO DOS ESTUDANTES
}

\begin{abstract}
BUILDING AND USING SYSTEMS OF CATEGORIES TO EVALUATE STUDENTS' UNDERSTANDING

ABSTRACT: We report in this paper three methodologies used to identify students' understanding levels when different kinds of research have been made. Systems of categories were built to analyze students' responses; these students were at different grades in High School. Our systems were elaborated under three perspectives: SOLO Taxonomy (BIGGS and COLLIS, 1982), Dynamic Skills Theory (FISCHER, 1980, 2006), and teacher's' views for evaluation. Although we reported two investigations, this paper aims to present three methods of qualitative analysis and to elicit criteria and procedures used to build the systems. We believe this kind of methodological issues must be discussed in educational research for improving methods, techniques and investigation parameters.

Keywords: Methodology, Science Teaching, Learning.

RESUMO: Neste trabalho, expomos três metodologias utilizadas para identificar patamares de entendimento de sujeitos em situações específicas de pesquisa. Construímos sistemas categóricos para analisar respostas de estudantes de diferentes séries do Ensino Médio. Três perspectivas fundamentaram a elaboração: a Teoria de Habilidades Dinâmicas (FISCHER, 1980, 2006), a Taxonomia SOLO (BIGGS e COLLIS, 1982) e a perspectiva docente de avaliação. Duas investigações são relatadas, porém, o foco do trabalho é evidenciar os três métodos de análise qualitativa, explicitando os critérios e os procedimentos realizados para a construção dos sistemas. Acreditamos que discussões metodológicas dessa natureza devam ser realizadas no âmbito das pesquisas educaciotermos de elaboração e aplicação.

Palavras-chave: Metodologia, Ensino de Ciências, Aprendizagem.
\end{abstract}

Amanda Amantes* Elrismar Oliveira**

*Doutora em Educação pela Universidade Federal de Minas

Gerais (UFMG). Professora

Adjunta do Departamento II da Faculdade de Educação da Bahia (UFBA).

E-mail:

amandaamantes@gmail.com

* * Mestre em Ensino de Física pela Pontifícia Universidade Católica de Minas Gerais (PUC. MG). Professora Assistente do Instituto Educação, Agricultura e Ambiente da Universidade Federal do Amazonas (UFMA). E-mail: elrismar@gmail.com 


\section{INTRODUCุ̃̃o}

Nos últimos anos, questões relativas à qualidade e ao rigor das investigações na área de ensino têm surgido como uma preocupação, principalmente no que se refere a aspectos metodológicos. Discussões sobre validade e confiabilidade, termos geralmente incorporados em pesquisas de cunho quantitativo, são levantadas no âmbito educacional para pontuar problemas relevantes em investigações que se baseiam na análise qualitativa de dados (GOLAFSHANI, 2003; GORARD, 2002; MORSE, 2002).

Um exemplo é o uso de técnicas desenvolvidas para um contexto específico em outras situações cujo problema de pesquisa não é condizente com tal método. Nesse sentido, a coerência interna da investigação muitas vezes é comprometida pela imposição de uma técnica e/ou referencial teórico que não são apropriados a questões, ao fenômeno ou ao problema a ser pesquisado. Gorard (2002) ressalta que em muitos casos métodos são utilizados por admiração, em vez de adequação.

Algumas discussões apontam para o fato de que é essencial estabelecer metodologias que incorporem as especificidades necessárias para atender aos objetivos de cada investigação, e isso implica em utilizar mais de um método quando necessário. A triangulação de dados e de técnicas, assim como a utilização de metodologias quali-quanti de análise, são maneiras consideradas válidas para garantir uma maior adequação e coerência nas pesquisas (SAGLAM-ARSLAN, 2010; GOLAFSHANI, 2003). Entretanto, essa coerência é conseguida por um esforço de construção de desenhos de pesquisa que consigam, ao mesmo tempo, conformar métodos, objetivos, referencial teórico, resultados e implicações. Esse processo compreende fases, desenhadas e planejadas de acordo com parâmetros, problemas e questões da pesquisa; Nesse sentido, "algumas delas se parecem mais como uma arte on grafite, e outras mais como Ciência” (GORARD, 2002, pág. 354).

Dentre as fases de desenho e planejamento, a de tratamento de dados envolve a tomada de decisão sobre os instrumentos e métodos que serão utilizados. Os dados usualmente coletados se referem a entrevistas, observações, respostas de questionários e gravações em áudio e vídeo, o que abarca muitas possibilidades de análise. A categorização de respostas, episódios, ações e outras unidades definidas em cada contexto de pesquisa é uma metodologia largamente utilizada. Entretanto, esse uso muitas vezes é problemático, pois nem sempre há coerência entre todos os aspectos da pesquisa (teoria, objetivos, problemas, questões e outros) além de os sistemas estarem, em alguns casos, inadequados à natureza dos dados a serem analisados.

Tendo em vista a importância da construção de sistemas de categorias mais adequados aos parâmetros de pesquisa na área de ensino, o presente trabalho tem como foco expor e discutir três diferentes sistemas elaborados para análise de respostas de estudantes fornecidas em questionários e atividades escritas. Um dos sistemas se refere à Taxonomia SOLO, desenvolvida pelos autores Biggs e Collis (1982) como ferramenta para construção de escalas de entendimento. Ou- 
tro método de classificação de respostas é o nível de explicitação, e o terceiro diz respeito a níveis ou patamares hierárquicos de entendimento, definidos a partir da visão docente de avaliação em conjunto com aspectos da Teoria de Habilidades Dinâmicas (FISCHER, 1980, 2006).

\section{Pesquisas}

Os sistemas de categorias relatados neste trabalho foram construídos a partir de perspectivas diferentes da unidade de análise, mas com o intuito semelhante de acessar formas diferenciadas de entendimento. Dois dos sistemas foram desenvolvidos para análise do mesmo banco de dados; nesse caso, a pesquisa teve como objetivo avaliar o entendimento de estudantes do Ensino Médio sobre os conceitos de Movimento Relativo e Referencial Inercial. A outra categorização foi construída para avaliar o entendimento sobre conteúdos diversos relacionados ao funcionamento da televisão.

\section{Sujeitos, contexto e objetivos}

Ambas as pesquisas descritas neste trabalho foram realizadas em uma mesma escola pública federal, mas em épocas distintas, em um intervalo de três anos. A escola se enquadra na classificação de profissionalizante, oferecendo cursos em patologia clínica, eletrônica, química e instrumentação. $\mathrm{O}$ ano é divido em trimestres e as aulas ocorrem no período diurno, no formato integral de estudos. Os alunos têm aulas teóricas e práticas de disciplinas típicas do Ensino Médio, além de disciplinas técnicas específicas da formação profissional. A avaliação é feita por meio de provas, trabalhos e tarefas de laboratório. O ingresso na instituição é feito por concurso público e por progressão do Ensino Fundamental para o Ensino Médio, a partir de uma escola de Ensino Fundamental, sendo ambas pertencentes à mesma Instituição Federal de Ensino Superior.

A primeira pesquisa contou com questionários respondidos por quatrocentos e dezessete estudantes, distribuídos no primeiro, segundo e terceiro anos do Ensino Médio. Esse questionário foi aplicado uma única vez e tinha como propósito identificar como esses estudantes concebiam os conceitos de Referencial Inercial e Movimento Relativo.

Participaram da segunda pesquisa somente os alunos do primeiro e do terceiro ano do Ensino Médio. Foram seis turmas do primeiro ano (cento e quarenta e sete alunos) e cinco turmas do terceiro (cento e treze alunos), num total de duzentos e sessenta participantes. Os dados analisados são as respostas desses alunos, obtidas em testes de conhecimento aplicados antes e depois do estudo de um material. O propósito foi identificar o entendimento sobre conteúdos diversos, exibidos antes e depois de uma instrução.

\section{Coleta dos dados e métodos de análise}

$\mathrm{Na}$ pesquisa sobre o entendimento de Movimento Relativo e Referencial Inercial, a coleta de dados ocorreu por meio da aplicação de um questio- 
nário, em um mesmo momento, a todas as turmas. O questionário continha questões em que os alunos eram indagados diretamente sobre sua concepção, questões em que deveriam interpretar uma situação para resolver um problema qualitativo e questões de interpretação de uma situação para responder verdadeiro ou falso a um conjunto de afirmações. Em suma, procuramos identificar o entendimento em termos de conceitualização e em termos procedimentais. No primeiro caso pelas respostas às questões diretas sobre o que entendiam pelos conceitos; no segundo, quando os alunos tinham que mobilizar seu entendimento para solucionar problemas específicos ou interpretar uma situação, como exemplificado pela figura 1.

Figura 1: Exemplo de questão de interpretação

\section{Questão 4: Observe o desenho ao lado e leia as pro- posições consideradas. A seguir, coloque $V$ para as sentenças verdadeiras e $F$ para as falsas:}

I - 0 desenho seguinte mostra um trem em movimento próximo a uma estação. Imagine que um vendedor caminha na plataforma. Dentro do trem uma criança corre em direção à frente dele. Considere que S1 é o sistema de referência fixo na plataforma e S2 o sistema fixo no trem.

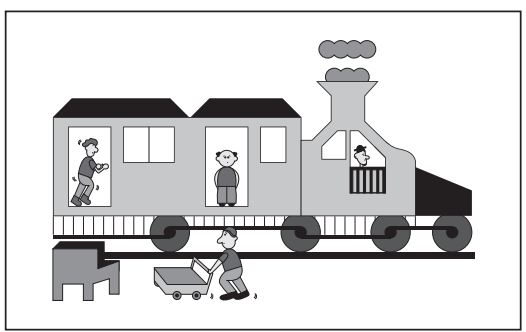

a) ( ) a criança está em movimento se visto de S1 e parada se visto de S2

b) ( ) a criança está parada se visto de S1 e o vendedor está em movimento se visto de S2

c) ( ) a criança está em movimento se visto de S1 e o vendedor está parado se visto de S2

d) ( ) a criança está em movimento se visto de S1 e o vendedor está em movimento se visto de S2

e) ( ) o vendedor realmente está em movimento

f) ( ) a criança realmente está em movimento

g) ( ) o vendedor está parado se visto de S1 e em movimento se visto de S2

As respostas das questões diretas foram classificadas segundo seu nível de explicitação (concepção acadêmica do conteúdo - Categorias de Explicitação) e em termos da estrutura do entendimento (Taxonomia SOLO).

A perspectiva da segunda pesquisa foi avaliar a aprendizagem a partir da mudança identificada para o entendimento quando este foi mensurado em diferentes momentos. Uma Unidade Temática sobre o funcionamento da televisão foi elaborada como material didático e utilizada em intervenção educacional no primeiro e terceiro anos. O estudo foi de aproximadamente três semanas, sendo a aplicação feita pelos próprios professores da disciplina. O caráter da Unidade foi interdisciplinar e contextual, dentro da perspectiva dos Parâmetros Curriculares Nacionais (BRASIL,1999). Ela contemplou conteúdos de Biologia, Química, Informática e Física, tendo como foco os princípios gerais de funcionamento e não o aparato em $\mathrm{si}^{1}$. A maior parte das atividades e tarefas estava em formato virtual. Dessa maneira, os alunos estudaram em grupo, contando cada grupo com um computador.

A coleta de dados foi feita mediante gravação em áudio de cada grupo, 
atividades escritas, testes de conhecimento aplicados antes e após o estudo da Unidade, entrevistas com alguns alunos e caderno de bordo. Diferentes análises foram feitas esses dados, tanto de natureza qualitativa como de quantitativa. Neste trabalho, iremos reportar a análise qualitativa realizada com os testes de conhecimento. Eles foram constituídos de três questões abertas, em que os estudantes deveriam descrever o seu entendimento sobre alguns conteúdos relacionados ao funcionamento da TV e duas questões com itens de verdadeiro e falso.

\section{Construção dos sistemas de categorias}

As dificuldades inerentes às pesquisas na área educacional estão muitas vezes associadas ao caráter subjetivo do objeto de estudo. No nosso caso, lidamos com a aprendizagem de conteúdos de alto nível de complexidade e, como dado, obtemos geralmente declarações verbais ou escritas.

Em nossa pesquisa, concebemos que houve aprendizagem quando identificamos um progresso no entendimento explicitado nessas declarações, ao longo de um determinado tempo. Para identificar esse progresso, é preciso estabelecer alguns critérios que nos permitam afirmar que o entendimento avaliado em determinado instante é, hierarquicamente, maior ou menor (ou pior e melhor, mais profundo e menos profundo) que o entendimento avaliado em outro momento. Nesse sentido, construímos escalas fundamentadas em teorias cognitivas para o desenvolvimento e na perspectiva docente de avaliação: Taxonomia SOLO e Categorias de Explicitação para a primeira investigação, e Categorias de Conteúdo para a segunda.

\section{Taxonomia SOLO}

Os autores Biggs e Collis (1982) desenvolveram uma Taxonomia denominada SOLO (Structure of Observing Learning Outcome) que pode ser utilizada como ferramenta em vários contextos, inclusive como metodologia de análise de dados em pesquisas qualitativas.

Esses autores admitem a existência de estágios de desenvolvimento cognitivo que, assim como na teoria piagetiana, surgem em idades aproximadamente definidas. Entretanto, diferente da teoria de Piaget, para Biggs e Collis o desempenho não depende necessariamente das capacidades cognitivas em certa fase da vida, ainda que seja delimitado pelas estruturas de pensamento que possuem em cada etapa. Eles concebem que um mesmo sujeito pode funcionar em vários estágios (ou modos, como denominados na teoria) simultaneamente, pois o desempenho está relacionado à área de conhecimento em que opera. A teoria que propõem é multimodal, e seu foco é na avaliação qualitativa da aprendizagem de conteúdos específicos. A forma de resolução de problemas em um determinado momento definirá os modos e não as estruturas cognitivas dos sujeitos.

Nessa perspectiva teórica, em cada domínio de ação passa-se de um estágio a outro por meio de um ciclo de aprendizagem que envolve uma progressão 
em níveis crescentes de complexidade do modo de funcionamento (AMANTES E BORGES, 2008). No modelo de Biggs e Collis, o ciclo de aprendizagem que leva de um estágio a outro possui cinco níveis de complexidade no modo de funcionamento do pensamento: pré-estrutural, uniestrutural, multiestrutural, relacional e abstrato estendido (BIGGS e COLLIS, 1982).

Esses níveis se relacionam tanto à qualidade como à quantidade de informações processadas. Eles podem ser descritos de forma abstrata e genérica, e relativamente a uma tarefa cognitiva que exija um modo específico de funcionamento (BIGGS e COLLIS, 1982, 1991). Assim, diferentemente de Piaget que concebe a ocorrência da mudança na forma do conhecimento quando o sujeito muda de estágio, para Biggs e Collis podem ocorrer evoluções no entendimento sem que haja essa mudança, ou seja, dentro de um mesmo modo pode haver um aumento da complexidade do entendimento.

A Taxonomia Solo tem sido usada de diferentes formas e em diferentes domínios de conhecimento: na avaliação de professores para identificar experts (BOND et al, 2000), para avaliar programas de ensino (MAGUIRE, 1988) e como instrumento metodológico de pesquisas educacionais (BIGGS, 1994; BIGGS, 1995; HAWKINS e HEDBERG, 1996; BIGGS E COLLIS, 1999; McMILLAN 2001; PANIZZON, 2003; HATTIE e BROWN, 2004; HOLMES, 2004; AMANTES e BORGES 2008). Como sugere uma progressão do entendimento em quatro níveis de complexidade dentro de um modo específico, sua utilização por professores tem implicado no desenvolvimento de programas para capacitar os estudantes a enriquecer e aumentar sua aprendizagem profunda (AMANTES, 2009). Em nosso trabalho, utilizamos a taxonomia para avaliar o entendimento que os estudantes demonstraram ao responderem as questões:

\section{0 que você entende por: \\ a) Referencial Inercial? b) Movimento Relativo?}

O objetivo da categorização foi verificar a concepção em relação aos conceitos por meio da identificação de elementos relevantes e pertinentes, assim como a maneira pela qual foram reportados e relacionados. A avaliação do entendimento foi feita a partir de duas perspectivas: i) se a ideia presente corresponde a elementos tangíveis e palpáveis ou a elementos mais abstratos e teóricos; e ii) qual o nível de relações entre os elementos reportados.

Como primeiro resultado, obtivemos uma classificação em dois modos: concreto simbólico e formal. Depois de classificada de acordo com o modo, as respostas foram categorizadas segundo seu nível de complexidade, estabelecido de acordo com a teoria: 
- Pré-estrutural (P): as respostas explicitadas são inadequadas. O indivíduo opera em modo aquém do que o solicitado na questão, sendo distraído ou confundido por aspectos irrelevantes pertencentes a um estágio ou modo prévio.

- Uniestrutural (U): o foco é correto, mas o aprendiz explicita poucas informações dos dados e as respostas podem ficar inconsistentes.

- Multiestrutural (M): o aprendiz se vale de características mais relevantes e corretas, mas elas não se integram totalmente; algumas inconsistências podem aparecer.

- Relacional (R): as informações são acessadas, os dados são avaliados e as relações são estabelecidas. O todo se torna uma estrutura coerente; não há inconsistências.

- Abstrato estendido (A): O aprendiz agora generaliza a estrutura para um novo quadro com características mais abstratas, representando um novo e elevado modo de operação. Normalmente esse nível torna-se o nível uniestrutural do modo seguinte da hierarquia de categorias de funcionamento.

Esse sistema, embora definidos os parâmetros de classificação a priori, foi construído a partir dos conteúdos investigados. Obtivemos, para o modo concreto simbólico, dois ciclos de níveis de complexidade. Isso pode ocorrer quando avaliamos conteúdos que compreendem muitos aspectos e apresentam um alto nível de complexidade. Os níveis são ordenados de acordo com um processo crescente de organização do número de dimensões relacionadas, de consistência entre essas relações e generalização dos princípios utilizados. $\mathrm{O}$ aumento da complexidade ocorre por uma crescente demanda pelo aumento da quantidade da memória de trabalho ou poder de concentração: nos níveis uni e multiestrutural, o estudante interpreta a informação dada e utiliza uma estratégia conhecida para fornecer a resposta, enquanto nos níveis relacional e abstrato estendido ele deve pensar em muitos objetos de uma só vez e avaliar quais são inter-relacionados (HATTIE e BROWN 2004). Um exemplo de definição das categorias pode ser visualizado no quadro 1.

A categorização foi validada por pares que tinham familiaridade com esse tipo de sistema. A Taxonomia SOLO constitui um bom instrumental para avaliar, de forma hierárquica, os níveis de complexidade de acordo com a acepção concreta e abstrata dos conceitos. Ela estabelece um sistema simples de categorias que independe do conteúdo e que pode ser aplicado como instrumento para diversos propósitos. Acreditamos que essa ferramenta atenda ao rigor que as pesquisas educacionais têm buscado, prestando-se a avaliar qualitativamente a aprendizagem dos estudantes, em diferentes conteúdos e de diferentes formas.

\section{Categorias de Explicitação}

Outro sistema para verificar o entendimento dos estudantes sobre Referencial Inercial e Movimento Relativo foi construído, tomando-se como parâmetro a perspectiva docente de avaliação. Nessa perspectiva, avaliamos a coerência e a correspondência do que é explicitado com a acepção acadêmica dos conceitos. 
Quadro 1: Definição das categorias de respostas de acordo com a

Taxonomia SOLO para o conceito de Referencial Inercial

\begin{tabular}{|c|c|c|}
\hline \multicolumn{3}{|c|}{ REFERENCIAL INERCIAL } \\
\hline \multicolumn{3}{|c|}{ MODO CONCRETO } \\
\hline \multicolumn{3}{|c|}{ Ciclo 1: o foco no movimento dos corpos } \\
\hline $\begin{array}{l}\text { U1: unidimensional; foco } \\
\text { no movimento dos corpos, } \\
\text { identificando o conceito de } \\
\text { referencial ao único objetivo } \\
\text { de análise desse movimento. } \\
\text { Ex: "Um objeto está em } \\
\text { movimento ou parado em } \\
\text { relação a outro corpo." }\end{array}$ & $\begin{array}{l}\text { M1: multidimensional; } \\
\text { foco no movimento, com } \\
\text { referência a ponto de vista } \\
\text { ou observação. } \\
\text { Ex: "Sempre que você for } \\
\text { olhar o movimento de algo } \\
\text { tem que se determinar } \\
\text { um referencial." }\end{array}$ & $\begin{array}{l}\text { R1: relacional; embora exista } \\
\text { grande coerência, o foco é no } \\
\text { conceito de referencial como } \\
\text { associado fortemente ao } \\
\text { movimento dos corpos. } \\
\text { Ex: "Sistema de referência } \\
\text { nos indica o "ponto" em que } \\
\text { estamos observando um } \\
\text { movimento, por ex com o } \\
\text { referencial na terra, podemos } \\
\text { dizer que o Sol gira em torno } \\
\text { da terra, mas se o referencial } \\
\text { for o Sol, dizemos que a terra } \\
\text { gira em torno dele." }\end{array}$ \\
\hline \multicolumn{3}{|l|}{ Ciclo 2: o foco no ponto de vista } \\
\hline $\begin{array}{l}\text { U2: compreensão sobre } \\
\text { sistema de referência mais } \\
\text { associada a ponto de vista, } \\
\text { mas são sucintas e com } \\
\text { foco único. } \\
\text { Ex: "É o ponto de vista de } \\
\text { uma pessoa." }\end{array}$ & $\begin{array}{l}\text { M2: incorporam novos } \\
\text { elementos à compreensão } \\
\text { de referencial como ponto de } \\
\text { vista, mas podem apresentar } \\
\text { certa incoerência. } \\
\text { Ex: "É ter algum ponto como } \\
\text { referência, para a partir } \\
\text { desse ponto observarmos os } \\
\text { fenômenos que ocorrem." }\end{array}$ & $\begin{array}{l}\text { R2: explicação mais } \\
\text { detalhada do conceito com } \\
\text { coerência interna. } \\
\text { Ex: "Sistema de referência } \\
\text { é o lugar onde observamos } \\
\text { certos fenômenos e neste lugar } \\
\text { estão incluídos pontos que } \\
\text { convenientemente julgamos } \\
\text { estar parados e pontos que } \\
\text { julgamos estar em movimento." }\end{array}$ \\
\hline \multicolumn{3}{|c|}{ MODO CONCRETO } \\
\hline $\begin{array}{l}\text { Uf1: compreensão em um } \\
\text { nível mais abstrato, mas } \\
\text { o foco é somente em um } \\
\text { ponto, seja relacionado a } \\
\text { movimento ou ponto de vista. } \\
\text { Ex:"Para mim, sistema de } \\
\text { referência é aquele ao qual } \\
\text { fazemos a observação de algo. } \\
\text { Referencial é o ponto do qual } \\
\text { observamos algo, portanto } \\
\text { este varia, pois podemos } \\
\text { observar algo de vários pontos } \\
\text { que nos permitem permitem } \\
\text { vários resultados diferentes." }\end{array}$ & $\begin{array}{l}\text { Mf1: compreensão mais } \\
\text { abstrata com novos } \\
\text { elementos, sem foco } \\
\text { específico; } \\
\text { Ex: "Referência é usada } \\
\text { para que o ponto de vista } \\
\text { seja avaliado, seja levado em } \\
\text { consideração. As respostas } \\
\text { de um problema podem ser } \\
\text { diferentes se mudarmos a } \\
\text { referência, o ponto de vista." }\end{array}$ & $\begin{array}{l}\text { Rf1: abstração mais elevada, } \\
\text { com incorporação de } \\
\text { elementos mais gerais, com } \\
\text { coerência interna e relação } \\
\text { entre os elementos. } \\
\text { Ex: "um ponto ou uma } \\
\text { área que se considera com } \\
\text { movimento e/ou energia nula } \\
\text { passando os outros corpos ou } \\
\text { partículas a ter movimento ou } \\
\text { energia quando comparado } \\
\text { a este." }\end{array}$ \\
\hline \multicolumn{3}{|c|}{$\begin{array}{l}\text { P: (Pré Estrutural): respostas não interpretáveis do ponto de vista do modo concreto-simbólico } \\
\text { ou do modo formal de pensamento. } \\
\text { Ex: "É um sistema circular"; "É uma observação física que tem referencial explicitado". "Sistema } \\
\text { que estuda as leis da mecânica visando pontos de vista diferentes." }\end{array}$} \\
\hline
\end{tabular}


Partimos do pressuposto de que a forma de explicitar corretamente um entendimento está associada a como o sujeito articula os elementos a ele relacionados. Isso implica que o entendimento sobre algo pode estar mais ou menos articulado, sendo sua explicitação na forma declarativa um indício do nível desse entendimento. A explicitação adequada incorpora linguagem, lógica e estrutura próprias de um conhecimento formal. . Entretanto, acreditamos que essa é uma das muitas formas de acesso ao entendimento e que ela, por si só, não é suficiente para alcançar todos os aspectos relevantes. Nossa postura é a de que o estudante demonstra um entendimento hierarquicamente mais elevado quando mobiliza um conteúdo de maneira coerente em termos procedimentais e de conceitualização (AMANTES, 2009).

O foco do sistema de explicitação está em avaliar a exatidão das respostas tendo-se como parâmetro as definições acadêmicas dos conceitos, o que geralmente é feito por professores em suas avaliações. Nesse sentido, procuramos trabalhar com as ideias centrais explicitadas como um todo, sem nos preocupar em identificar e avaliar os elementos e suas relações de maneira sistemática. Esse tipo de categorização é semelhante ao utilizado por Saglam-Arslan (2010) para classificar o entendimento de estudantes sobre o conceito de energia. As categorias foram três:

- $\boldsymbol{E}$ - explícitas: as ideias se apresentam de forma articulada e de fácil entendimento, além de estarem condizentes com a definição cientificamente aceita. As respostas são facilmente compreendidas pelo leitor, traduzem claramente o entendimento correto sobre os conceitos.

- PE - parcialmente explícitas: a ideia não é muito clara, mas as respostas possuem indícios de que o entendimento é correto; elas têm elementos coerentes, mas não estão totalmente condizentes com a definição acadêmica, pois podem apresentar alguns equívocos (que não comprometem sua aceitação parcial).

- NE - não explícitas: as respostas são vagas, não se referindo de maneira objetiva ao que foi perguntado. Se apresentam equivocadas para os padrões acadêmicos e não é possível identificar com clareza o entendimento do estudante.

O quadro 2 apresenta exemplos de respostas categorizadas segundo esse sistema. 
Quadro 2: Respostas para o conceito de Sistema de Referência, categorizadas segundo o sistema de explicitação. NE- não explicita/ PE- parcialmente explicita/ E- explicita.

\begin{tabular}{|l|l|}
\hline RESPOSTA & DESCRIÇÃO DA CATEGORIA \\
\hline "É um sistema que possui um referencial." & $\begin{array}{l}\text { NE: Não é possível identificar o } \\
\text { entendimento do aluno, uma vez que as } \\
\text { informações estão dispostas de maneira a } \\
\text { impedir a compreensão da ideia formulada. } \\
\text { Não transmitem ideia alguma, pois são } \\
\text { desprovidas de contexto, tornando-se sem } \\
\text { sentido quando interpretadas pelo leitor. }\end{array}$ \\
\hline $\begin{array}{l}\text { "O movimento de um corpo depende do } \\
\text { sistema a sua volta, pois ele pode estar se } \\
\text { movimentando em relação a um outro corpo } \\
\text { e estar parado em relação a um terceiro. O } \\
\text { sistema de referência é o sistema de sua volta." }\end{array}$ & $\begin{array}{l}\text { PE: Há poucas evidências sobre o que o } \\
\text { estudante entende por Sistema de Refência, } \\
\text { mas podemos, ainda assim, inferir algo } \\
\text { sobre sua concepção, como sendo pautada } \\
\text { no conceito como ponto de vista ou como } \\
\text { objetos concretos que servem de referência, } \\
\text { principalmente ao movimento. Pela } \\
\text { explicação não sabemos se o aluno entende } \\
\text { o conceito em termos de objetos a sua volta, } \\
\text { mas certamente a sua ideia é concreta, uma } \\
\text { vez que relaciona movimento a um corpo } \\
\text { que serve como referência; identificamos, } \\
\text { então, elementos inadequados ("O sistema de } \\
\text { referência é o sistema de sua volta") ao mesmo } \\
\text { tempo em que temos um indício de que sua } \\
\text { concepção esteja razoavelmente aceitável. }\end{array}$ \\
\hline $\begin{array}{l}\text { "Para mim , a expressão'sistema de referência' } \\
\text { significa a relação usada para comparação } \\
\text { de grandezas diferentes. Assim, há infinitos } \\
\text { sistemas de referências, tanto na matemática } \\
\text { quanto na física, que são usados em medidas, } \\
\text { distância, pontos referencias e etc..." }\end{array}$ & $\begin{array}{l}\text { E: O entendimento é expresso de forma } \\
\text { clara, possibilitando ao leitor identificar a } \\
\text { maneira pela qual o estudante concebe o } \\
\text { tema da questão. Nessa resposta, a definição } \\
\text { do termo passa pela consideração de que ele } \\
\text { representa um ponto de vista, perspectiva a } \\
\text { partir da qual um fenômeno é observado. }\end{array}$ \\
\hline
\end{tabular}

Podemos dizer que, de uma forma geral, as Categorias de Explicitação se constituem como um sistema de classificação que pode ser facilmente incorporado em ações docentes, pois sua lógica é semelhante à que os professores usam para corrigirem atividades dos alunos. Essa lógica não requer conhecimento sobre teorias de aprendizagem, uma vez que se fundamenta em julgamento de exatidão e coerência com os padrões acadêmicos. Nesse sentido, o sistema não permite fazer maiores conjecturas sobre o entendimento a não ser como apropriação adequada da linguagem científica e como articulação, em nível de explicitação, das ideias academicamente válidas.

O valor das Categorias de Explicitação está justamente em nos fornecer mais uma pista do estado de articulação do entendimento. Na perspectiva adotada para este trabalho, a apropriação da linguagem acadêmica de forma a explicitar coerentemente a ideia correta sobre os conteúdos é um indício de que o estudante tem um entendimento mais articulado, embora não possamos, por esse sistema, delimitar qual o nível dessa 
articulação. Essas categorias são mais simples e diretas do que as outras reportadas, mas são importantes no sentido de propiciar discussões acerca da equiparação dos parâmetros de avaliação docente com outros parâmetros de avaliação do entendimento, além de nos fornecer uma dimensão a mais para investigar características do dado observado.

\section{Categorias de Conteúdo}

O objetivo principal da segunda pesquisa relatada neste trabalho foi investigar a aprendizagem de conteúdos estudados na Unidade Temática sobre o funcionamento da TV. Para tanto, um parâmetro de comparação entre os entendimentos exibidos ao longo do tempo foi definido, estabelecido por um sistema de categorização que nos permitisse identificar, em uma escala hierárquica, o entendimento sobre conteúdos. As respostas avaliadas foram dadas às seguintes questões do teste de conhecimento:

1. Explicite seu entendimento sobre o funcionamento da TV do ponto de vista da tecnologia e dos fenômenos físicos envolvidos.

2. Compare a TV que utiliza como princípio de funcionamento à tecnologia digital e à TV que funciona a partir da tecnologia analógica.

3. Diga o que você sabe sobre o funcionamento da TV de tubos de Raios Catódicos e a TV de Plasma, tentando explicitar as diferenças entre elas.

Incialmente utilizamos a Taxonomia SOLO como ferramenta. Entretanto, nos deparamos com a impossibilidade de adequar esse sistema ao tipo de questões propostas na perspectiva de avaliação de aprendizagem da segunda pesquisa. Isso porque as categorias obtidas, utilizando-se a taxonomia, são mais restritas e dizem respeito a conteúdos bem delimitados nas perguntas, sendo mais apropriadas para categorizar dados obtidos a partir de questões bem direcionadas, o que não foi o caso da segunda investigação.

O sistema que utilizamos foi o de Categorias de Conteúdo, estabelecido na perspectiva de avaliação docente de um conteúdo, mas com um refinamento de níveis hierárquicos de complexidade do entendimento. Essa complexidade é definida por patamares que subentendem uma interpretação teórica de desenvolvimento: a concepção é a de que um entendimento vai se constituindo por um caminho demarcado por diferentes fatores, dentre eles a familiaridade com o tema, o suporte social, o estado emocional e cognitivo, a maturidade, dentre outros (FISCHER, 1980). Nesse caminho, habilidades vão sendo integradas, associadas e construídas, estando diretamente relacionadas à estruturação de conhecimentos em níveis hierárquicos ascendentes. Essa é a perspectiva da Teoria de Habilidades Dinâmicas (FISCHER, 1980, 2006), que como a Teoria SOLO postula estágios de desenvolvimento em um domínio específico de conhecimento, mas que, diferentemente dela, concebe a variação individual como determinante para explicar a passagem de um estágio a outro.

As Categorias de Conteúdo, apesar de incorporarem a ideia de níveis hierárquicos da Teoria de Habilidades Dinâmicas, não delimita estágios. Dawson (2004) constrói um sistema semelhante ao que fizemos para análise do conteúdo de Energia, fundamentado na mesma teoria. Essa autora descreve seu sistema como uma ferramenta denominada Lectical Assessment System (LAS), por meio da qual o entendimento é avaliado de acordo com a quantidade de conceitos e/ou conteúdos explicitados pelos estudantes 
em suas respostas. Outros pesquisadores utilizam ferramentas semelhantes para analisar o entendimento de conceitos, como Rappolt-Schlichtmann et al (2007), que apresentam categorias sobre o conteúdo de densidade e analisam tarefas para identificar patamares de desenvolvimento de acordo com a Teoria de Habilidades Dinâmicas.

As categorias do sistema elaborado compreendem dois aspectos: os conteúdos a que se referem e o nível de complexidade com o qual esses conteúdos podem ser avaliados. Os conteúdos ou conceitos levantados foram considerados como temas. Assim, para uma mesma questão, tivemos diferentes temas que puderam ser constatados nas respostas. Além da identificação dos temas, o sistema compreendeu, ainda, uma forma de avaliar a estrutura da resposta de acordo com o número de elementos incorporados, sua natureza e as relações estabelecidas. Também para esse sistema, foram definidos níveis hierárquicos de complexidade a partir da natureza de conteúdo reportado e a forma como a lógica entre os elementos foi estabelecida.

Como exemplo, podemos analisar o quadro 03. Nele, é representado um sistema construído especificamente para determinada questão. A partir da leitura das respostas, delimitamos seis temas que dizem respeito ao conteúdo que podem ser reportados pelos estudantes. Para cada tema, o estudante pode exibir um entendimento mais ou menos estruturado, o que é caracterizado pelas categorias que correspondem aos níveis hierárquicos de entendimento. Esse procedimento foi realizado para as três questões abertas. A classificação das respostas, segundo esse sistema, nos possibilitou obter 51 itens a partir de três questões (considerando os níveis de complexidade). Esse procedimento permitiu que fizéssemos um tratamento estatístico dos dados para investigar a mudança de um instante de medida a outro.

É importante ressaltar um aspecto que diferencia esse sistema dos outros descritos anteriormente: ele se pauta tanto em teoria como nos dados. A teoria determina a construção dos níveis hierárquicos, mas esses só são definidos a partir dos temas e conteúdos identificados nas respostas dos estudantes. Portanto, temos, a princípio, uma estrutura que orienta e direciona o olhar para os observáveis e que, posteriormente, determina a escala qualitativa de avaliação.

Os três sistemas descritos foram utilizados em duas pesquisas diferentes que tinham em comum o objetivo de avaliar o entendimento exibido por estudantes quando este foi verbalizado por escrito. Apesar de os propósitos terem sido semelhantes, o foco de avaliação do entendimento foi diferente, pois houve distinção em relação à consideração da dimensão do construto.

Assim, os sistemas utilizados como instrumentos de categorização se diferem em termos de concepção teórica e em termos de elaboração. O primeiro se baseia fundamentalmente em pressupostos pré-estabelecidos teoricamente; o segundo foi construído em meio à leitura dos dados e a partir de uma visão docente apenas, enquanto o último foi estruturado por uma coordenação entre a acepção acadêmica (perspectiva docente de avaliação), perspectivas teóricas do desenvolvimento cognitivo e leitura dos dados. Embora abordem diferentes características que compõem um atributo de difícil acesso como o entendimento, esses sistemas nos fornecem parâmetros gerais para que vários aspectos sejam analisados em conjunto. 
Quadro 3: A delimitação das células não está clara, o que confunde a percepção das delimitações e descrições das categorias

\begin{tabular}{|c|c|c|}
\hline Temas & Definição das categorias & Níveis \\
\hline $\begin{array}{l}\text { Captura da } \\
\text { informação }\end{array}$ & $\begin{array}{l}0 \text { som e a imagem são “capturados" por dispositivos específicos. Reconhecem a existência do microfone e/ou câmera para captar o fenômeno a ser transformado em corrente. } \\
0 \text { som e a imagem são capturados por dispositivos específicos e transformados em sinal elétrico. } \\
\text { Há o reconhecimento de que o som e a imagem são captados por dispositivos específicos com a intenção de serem transformados em corrente elétrica. } \\
0 \text { som e a imagem são capturados e transformados em corrente elétrica variável, que carrega a informação. } \\
\text { Nesse tipo de resposta é explicitada a idéia de que a corrente correspondente à informação captada tem caráter variável. }\end{array}$ & $\begin{array}{l}\text { PE1C1 } \\
\text { PE1C2 } \\
\text { PE1C3 }\end{array}$ \\
\hline $\begin{array}{l}\text { Digitalização } \\
\text { da informação }\end{array}$ & $\begin{array}{l}0 \text { sinal elétrico é digitalizado para a transmissão digital. Só reconhece que o sinal digitalizado, sem fazer referência ao código binário ou aos processos. } \\
0 \text { sinal elétrico é codificado em bits/números binários para a transmissão digital. Menciona que na digitalização, a informação é codificada em bits ou números binários. } \\
0 \text { sinal elétrico passa por processo de amostragem, quantização e codificação para a transmissão digital. } \\
\text { Menciona os processos através dos quais a informação é transformada em códigos binários. }\end{array}$ & $\begin{array}{l}\text { PE1D1 } \\
\text { PE1D2 } \\
\text { PE1D3 }\end{array}$ \\
\hline $\begin{array}{l}\text { Meio de } \\
\text { transmissão } \\
\text { da informação }\end{array}$ & $\begin{array}{l}\text { A informação e transmitida por ondas eletromagnéticas. Reconhece o meio de transmissão das informações como sendo através de ondas eletromagnéticas. } \\
\text { A informação é transmitida por ondas eletromagnéticas, cabo ou satélite. Reconhece outros meios de transmissão, além das ondas eletromagnéticas: cabo e satélite. } \\
\text { A informação do som e imagem é transmitida separadamente, por ondas eletromagnéticas/cabo/satélite. } \\
\text { Reconhece que a informação sobre a imagem é transmitida separadamente da informação sobre o som, além de mencionar o meio de transmissão. } \\
\text { As ondas eletromagnéticas/sinal elétrico que transmitem a informação são modulados por outro sinal, que carrega a informação. } \\
\text { Menciona que para serem transmitidas, as informações modulam a onda portadora. }\end{array}$ & $\begin{array}{l}\text { PE1T1 } \\
\text { PE1T2 } \\
\text { PE1T3 } \\
\text { PE1T4 }\end{array}$ \\
\hline Antenas & $\begin{array}{l}\text { Os elétrons da antena transmissora oscilam e produzem onda eletromagnética, que carrega a informação. } \\
\text { Reconhecem a oscilação dos elétrons como fenômeno responsável pela produção das ondas eletromagnéticas. } \\
\text { Os elétrons da antena receptora oscilam de acordo com a onda eletromagnética que traz a informação. } \\
\text { Reconhecem a correspondência entre a informação sobre o som e imagem e a forma como os elétrons oscilam e produzem ondas eletromagnéticas. } \\
\text { Os elétrons da antena transmissora oscilam e produzem onda eletromagnética de mesma freqüência, que carrega a informação e faz os elétrons da antena } \\
\text { receptora oscilarem também na mesma freqüência. } \\
\text { Descrevem todo o processo de oscilação da antena transmissora, de acordo com a informação, passando pela produção de onda eletromagnética até a oscilação } \\
\text { novamente dos elétrons da antena receptora, com mesma freqüência. }\end{array}$ & PE1A1 \\
\hline $\begin{array}{l}\text { Recepção da } \\
\text { informação e } \\
\text { interpretação } \\
\text { dos sinais }\end{array}$ & $\begin{array}{l}\text { A informação é interpretada e transformada em imagem e som. Menciona somente que a informação recebida é interpretada para ser transformada em som e } \\
\text { imagem novamente } \\
\text { A informação é transformada em corrente elétrica; depois de recebida, é convertida em imagem e som. } \\
\text { Reconhecem que a informação passa pela transformação em corrente elétrica, antes de ser transmitida e depois de recebida. } \\
\text { Os sinais são interpretados e transformados em som e imagem; se estiverem na forma digital, são decodificados. } \\
\text { Mencionam a questão da codificação e decodificação, reconhecendo que os sinais podem estar na forma digital. }\end{array}$ & $\begin{array}{l}\text { PE1R1 } \\
\text { PE1R2 }\end{array}$ \\
\hline Aparelho & $\begin{array}{l}\text { A TV de TRC tem um tubo ou canhão de elétrons. A TV de plasma tem um gás. } \\
\text { Expressam os elementos mais marcantes que estão presentes nos dois tipos de aparelho, que dão o nome a eles. } \\
\text { Na TV de TRC há uma varredura da tela por um feixe de elétrons. Na TV de plasma há uma varredura pela variação de potencial nas microlâmpadas. } \\
\text { Reconhecem como a imagem é formada na tela dos aparatos, além de citar seus componentes. } \\
\text { Na TV de TRC a tela é varrida por um feixe de elétrons que estimulam pontos cobertos por material fotossensível. A ddp na TV de plasma provoca a ionização do } \\
\text { gás que estimula o material fotossensível que cobre a tela. Sofisticam mais a resposta fornecendo informação sobre como a imagem é formada na tela, fazendo } \\
\text { referência aos elementos presentes em cada uma e aqueles que são essenciais. }\end{array}$ & $\begin{array}{l}\text { PE1Ap1 } \\
\text { PE1Ap2 } \\
\text { PE1Ap3 }\end{array}$ \\
\hline
\end{tabular}




\section{SÍNTESE DOS RESULTADOS}

Nas duas investigações conduzidas, constatamos patamares diferenciados de entendimento para os alunos das três séries, de acordo com cada sistema adotado. Na primeira pesquisa, os alunos que tiveram contato com o tema mais de uma vez e em tempo recente apresentaram um entendimento mais estruturado do ponto de vista acadêmico (alunos do terceiro ano, seguidos dos alunos do primeiro ano e, depois, do segundo).

$\mathrm{Na}$ segunda investigação houve aprendizagem, tanto para os alunos do primeiro ano como para os do terceiro, mas ela apresentou perfis diferenciados: os do terceiro ano tiveram maior progresso em tarefas do domínio tecnológico de conhecimento, enquanto que para os do primeiro ano isso ocorreu para conteúdos do domínio científico-escolar.

Esses resultados se baseiam na perspectiva teórica de que um crescimento do nível de entendimento implica em aprendizagem. Portanto, encontrar evidências de um grau ascendente de complexidade e exatidão dos conteúdos, tanto em termos de conceitualização como em termos procedimentais (evidenciado em tarefas específicas) indicam aprendizagem. Em cada sistema elaborado o intuito foi o de gerar um parâmetro de comparação do entendimento do ponto de vista descritivo.

Para a categorização pela Taxonomia SOLO, as interpretações e os resultados nos apontam para o nível de formalização de pensamento dos estudantes, em termos dos parâmetros da teoria. Em linhas gerais, constatamos que os alunos das três séries operam no modo Concreto-Simbólico para os dois conceitos, sendo a porcentagem maior para o conceito de Movimento Relativo. Verificamos, ainda, que mesmo que a maioria se concentre no modo Concreto-Simbólico, os alunos da terceira série possuem um entendimento mais complexo dos conceitos, pois apresentaram maior porcentagem nos modos mais altos de funcionamento detectados: Concreto-Simbólico 2 e Formal, tanto para o conceito de Movimento Relativo como para o de Referencial Inercial.

Para o segundo sistema de categorias, as de explicitação, fizemos a análise baseada na diferença entre séries. Verificamos que para o conceito mais abstrato não há muita diferença entre os alunos na forma de explicitação, mas há maior porcentagem de alunos do terceiro ano que conseguiram explicitar melhor seu entendimento em relação aos alunos de primeiro ano, cujo contato tanto com a disciplina de Física, como com o conteúdo, era inicial2. Os alunos da segunda série não tiveram índices muito superiores aos da primeira, demonstrando que em grande parte estão no mesmo patamar. Em linhas gerais, constatamos que os alunos da terceira série apresentaram um entendimento melhor estruturado que os alunos da primeira e segunda. Verificamos que esses alunos demonstraram maior habilidade em explicitar conceitos mais abstratos e que, quanto ao nível de formalização, parecem superar os das outras séries.

Na segunda pesquisa, as categorias de conteúdo se constituíram em dados de segunda ordem, utilizados em outras análises. Elas foram os instrumentos que 
permitiram obter uma escala qualitativa para o entendimento, o qual foi avaliado em momentos distintos da pesquisa. Os resultados obtidos se referem a uma análise mais complexa, sendo as categorias não reportadas diretamente. A partir dos temas e níveis de complexidade do sistema categórico utilizado, obtivemos uma escala do tipo Guttman ${ }^{3}$ (1944). Transformados em dicotômicos, os dados categorizados foram analisados a partir de parâmetros estatísticos. Investigamos como mudou o entendimento de um instante a outro a partir da escala construída qualitativamente.

Nossos resultados mostraram que os alunos, tanto da primeira como da terceira séries, progrediram de forma diferenciada e apresentaram perfis de entendimento distintos, independente da série. Isso significa que a Unidade Temática promoveu aprendizagem de naturezas diversificadas, um resultado que corrobora o fato de que é impossível promover o mesmo caminho de desenvolvimento a sujeitos submetidos a uma mesma instrução. Fatores como o engajamento e interesse mudaram o foco de atenção. Constatamos, ainda, que a aprendizagem foi maior para conteúdos pouco familiares. A frequência de abordagem e a natureza descritiva da categoria foram fatores que explicaram o progresso no entendimento.

As categorias de conteúdo foram incorporadas na pesquisa de uma maneira distinta dos outros dois sistemas, sendo o fundamento para análise quantitativa. Entretanto, ela teve o mesmo papel de estabelecer critérios bem específicos de avaliação do entendimento na perspectiva qualitativa, como nos outros casos. Isso mostra que outras possibilidades podem ser concebidas em relação ao tratamento de dados, como a integração de uma análise categórica a procedimentos quantitativos. Isso deve ser feito com o cuidado de preservar a dimensão de cada análise, atendendo sempre ao princípio da coerência interna.

\section{Categorização como ferramenta metodológica}

Tendo em vista a relevância dos métodos empregados para analisar os dados no âmbito da pesquisa educacional, devemos discutir como fundamentar e promover metodologias que melhor atendam a um rigor próprio das investigações acadêmicas. Uma forma de buscarmos aprimorar nossos métodos é estabelecer critérios mais precisos de categorização de dados.

No presente relato, foram explicitados três processos de elaboração de sistemas de categorias para investigar como os estudantes entendem conteúdos de Ciências e de outros domínios. O pressuposto geral que fundamentou as duas pesquisas é o de que a aprendizagem subentende fases em que o entendimento se aprofunda ao longo do tempo, influenciado por fatores diversos, como aspectos emocionais, socioculturais e a própria história de aprendizagem (FISCHER, 2006, AMANTES, 2009). Outro pressuposto é que temos acesso a indícios do estado de articulação desse entendimento que, por sua vez, pode ser expresso por diferentes habilidades, tanto no domínio da conceitualização, como em aspectos procedimentais. Esses indícios, quando investigados em conjunto, possibilitam compreender aspectos essenciais do progresso do entendimento e nos fornece elementos mais contundentes para interpretar o processo de aprendizagem. 
Nessa perspectiva, identificar o entendimento em suas diferentes manifestações é um caminho promissor do ponto de vista metodológico. Para tanto, é preciso que se desenvolva métodos e técnicas de análise coerentes e válidos para conduzir uma investigação dessa natureza. Coerentes porque devem atender à dimensão do entendimento avaliado, mantendo os limites e as possibilidades de sua aplicação para cada especificidade desse construto. Válidos no sentido de atenderem aos propósitos, hipóteses, pressupostos e referencial teórico da pesquisa conduzida.

A decisão em se utilizar os três sistemas para avaliar um mesmo objeto o entendimento - foi tomada em virtude dos distintos focos de investigação, das diferentes hipóteses levantadas para cada uma delas e, principalmente, da natureza complexa do objeto. A definição do que seja entendimento é ampla e polifocal (BORGES e AMANTES, 2003), por isso sua investigação não deve se restringir a uma única forma ou a um único método. Por outro lado, não podemos nos valer de uma técnica que se abrevia à aplicação em um único contexto específico de pesquisa, pois podemos incorrer no erro de construir artefatos metodológicos que corroborem resultados esperados. O mais importante é que haja adequação do propósito ao método (GORAD, 2002), mantendo-se a coerência interna de toda a pesquisa.

Devemos nos preocupar em construir instrumentos cujas características são mais gerais (podem ser aplicados em outras circunstâncias), mas que, ao mesmo tempo, sejam bem definidos em termos da dimensão, domínio ou especificidade do construto. Além disso, esses instrumentos devem ser estabelecidos por critérios que garantam uma definição e posição igualmente claras do ponto de vista teórico.

Muitas pesquisas na área de educação têm procurado desenvolver sistemas de categorização dessa natureza, a fim de estabelecer um maior rigor metodológico e obter resultados mais robustos. Coelho e Borges (2010), Dawson (2008), Rappolt-Schlichtmann (2007), Hattie, (2004), Holmes (2005), Kennedy (2005), Schwartz e Fischer (2004), Pannizon (2003), dentre outros, são alguns dos pesquisadores que têm se dedicado a elaborar e aperfeiçoar metodologias de classificação de concepções, entendimento, habilidades e outros atributos latentes.

Tal procedimento é negligenciado e/ou considerado menos importante em muitas pesquisas na área de ensino. Defendemos que, apesar de demandar esforço e tempo considerável em uma perspectiva de trabalho acadêmico, esse processo garante, do ponto de vista metodológico, um aprimoramento no que se refere à construção de evidências. Uma vez respeitados os fundamentos aos quais se propõe, um sistema categórico bem elaborado se torna poderoso para interpretar dados e sistematizar as análises. Diferentes métodos podem ser utilizados como triangulação, assim como resultados gerados pela categorização podem ser utilizados como dados de segunda ordem para outras análises.

Enfim, a construção de sistemas categóricos mais elaborados, do ponto de vista teórico e metodológico, possibilitam não só um maior rigor e objetividade para análise de unidades qualitativas como também são promissores para serem explorados em outros contextos de pesquisa. Eles garantem maior clareza dos parâmetros de investigação e consistência dos resultados e implicações. 


\section{CONSIDERACÕ̃ES FINAIS}

Acreditamos que uma proposta que busque adequar e aproximar diferentes sistemas de categorias, principalmente com naturezas distintas como os aqui descritos, possam contribuir para a construção de melhores instrumentos para conduzir pesquisas no âmbito educacional.

Os sistemas de categorias relatados neste trabalho foram construídos no intuito de começar a explorar as possibilidades de tratamento de unidades de análise usualmente incorporadas em pesquisas com enfoque metodológico qualitativo. Eles se constituem em instrumentos exploratórios, elaborados com uma preocupação de atender à coerência interna das investigações conduzidas. Apesar de não terem sido aplicados em outros contextos, muito esforço foi demandado no processo de construção, o que possibilitou uma aprendizagem significativa do ponto de vista da técnica e expertise.

É preciso que reconheçamos a importância das escolhas dos métodos de análise dos dados como essenciais para fundamentar nossas investigações. Sistemas de categorias utilizados para análise não devem ser arbitrários, e sim apoiados em teorias subjacentes à pesquisa, assim como em pressupostos bem definidos e metodologias adequadas, o que confere coerência ao processo investigativo.

Um estudo e aprofundamento sobre a elaboração desses sistemas é profícuo no sentido de estabelecer relações, se possível, entre os diferentes sistemas, ressalvando a natureza de cada um. Podemos desenvolver instrumentos e métodos que possam ser aplicados em conjunto ou separadamente, dependendo do objeto e do objetivo da investigação. Com isso, estaremos contribuindo para que a metodologia na pesquisa em educação seja melhor discutida, para que nossas práticas sejam aprimoradas e para que nossos resultados sejam mais refinados. O intuito principal é o de buscar, cada vez mais, o rigor e a qualidade.

Agradecemos a grande colaboração do Dr. Oto Borges e ao apoio do CNPq.

\section{NOTAS}

${ }^{1}$ A abordagem no material é geral, permitindo ao estudante aprender os conteúdos como novidade ou aprofundar seu entendimento quando o assunto lhe é familiar. O objetivo foi manter o foco no princípio de funcionamento, e não utilizar o aparato para explicar fenômenos físicos específicos. O conteúdo foi abordado do ponto de vista dos aspectos tecnológicos e dos fenômenos físicos envolvidos no processo desde a captura da imagem e do som, passando pelo seu transporte até a recepção e a reprodução dos sinais.

${ }^{2}$ Os alunos do primeiro ano tinham estudado recentemente o tema, já os alunos do segundo ano tiveram contato somente no ano anterior, quando cursaram a primeira série. Os alunos do terceiro ano tiveram contato com os conteúdos no primeiro, segundo e terceiro anos, de forma explícita ou subjacente a outros conteúdos, uma vez que o currículo adotado para a disciplina de Física na insti- 
tuição pesquisada é no formato recursivo (espiral).

${ }^{3}$ A escala Guttman compreende uma hierarquia em que o último item ou alternativa possui uma dimensão mais complexa que abarca os itens ou alternativas anteriores. Acertar uma última alternativa ou pertencer à ultima categoria, como no caso deste trabalho, significa possuir o entendimento de todas as alternativas e categorias anteriores.

\section{REFERÊNCIAS BIBLIOGRÁFICAS}

AMANTES, A. O entendimento de estudantes do Ensino Médio sobre Movimento Relativo e Referencial Inercial. 2005. 183f. Dissertação (Mestrado) - Faculdade, Universidade Federal de Minas Gerais, Belo Horizonte, 2005. AMANTES, A. Contextualização no ensino de Física: feito sobre a evolução do entendimento dos estudantes. Orientador: Oto Borges. 2009. 275f. Tese (Doutorado) - Faculdade, Universidade Federal de Minas Gerais, Belo Horizonte, 2009.

AMANTES, A.; BORGES, O. Uso da taxonomia SOLO como ferramenta metodológica na pesquisa educacional. In: ENCONTRO NACIONAL DE PESQUISA EM EDUCAÇÃO EM CIÊNCIAS, 6, 2008, Florianópolis. Anais... Belo Horizonte: FAE\UFMG, v. Único. p. 1-12, 2008.

BIGGS, J.; COLLIS, K. Evaluating the quality of learning. the SOLO taxonomy. New York: Academic Press, 1982. BIGGS, J.; COLLIS, K. Multimodal Learning and the quality of intelligent behavior. In: ROWE, Helga A. H. (Ed.). Intelligence: reconceptualization and measurement. Hillsdale, NJ: Lawrence Erlbaum, 1991. Cap. 5, p.57-76.

BIGGS, J. Assessing for learning: Some dimensions underlying new approaches to educational assessment. The Alberta Journal of Educational Research, Edmonton, v.41, n. 1, p. 1-17, 1995.

BOND, L. et al. The certification system of the National Board for Professional Teaching Standards: a construct and consequential validity study (Research Report). Greensboro, NC: University of North Carolina at Greensboro, Center for Educational Research and Evaluation, 2000.

BORGES, Oto; AMANTES, Amanda. O Entendimento de estudantes do ensino médio sobre Sistema de Referência e Movimento Relativo. In: ENCONTRO NACIONAL DE PESQUISADORES ENSINO DE CIÊNCIAS, 4, 2003, Bauru, SP. Anais... Bauru, SP: [ABRAPEC], 2003.

BRASIL. Ministério da Educação. Secretaria de Educação Média e Tecnológica. Parâmetros Curriculares Nacionais: ensino médio. Brasília: MEC, 1999.

COELHO, Geide R.; BORGES, Oto. O Entendimento dos estudantes sobre a natureza da luz em um currículo recursivo. Caderno Brasileiro de Ensino de Física, Florianópolis, v. 27, n. 1, p. 63-87, abr. 2010.

DAWSON, T. L.; WILSON, M. The LAAS: A computerizes developmental scoring system for small - and large-scale assessments. Educational Assessment, v. 9, p. 153-191, 2004.

DAWSON, Theo L.; STEIN, Z. Cycles of research and application in education: Learning pathways for energy concepts. Mind, Brain, e Education, Cambridge, v. 2, n. 2, p. 90-103, 2008.

FISCHER, K. W. A theory of cognitive development: the control and construction of hierarchies of skills. Psychological Review, v. 87, p. 477-531, 1980.

FISCHER, K. W. Dynamic cycles of Cognitive and Brain development. In: BATTRO, A. M.; FISCHER, K. W. (Eds.). The educated brain. Cambridge, U.K.: Cambridge University Press. 2006.

GOLAFSHANI, N. Understanding reliability and validity in qualitative research. The Qualitative Report, Canadá, v. 8, n. 4, p. 597-607, 2003.

GORARD, Stephen. Can we overcome the methodological schism? Four models for combining qualitative and quantitative evidence. Research Papers in Education, v.17, n.4, p.345-361, 2002.

GUTTMAN, L. A basis for scaling qualitative data. American Sociological Review, n. 9, v. 2, p. 139-150, 1944. HAWKINS, Wayne; HEDBERG. John G. Evaluating LOGO: use of the SOLO Taxonomy. Australian Journal of Educational Technology. Australia, v. 2, n. 2, p. 103-109, 1986.

HATTIE, J.A.C., \& BROWN, G.T.L. Cognitive processes in asTTle: The SOLO taxonomy. asTTTle Technical Report, University of Auckland/Ministry of Education, n. 43, set. 2004. 
HOLMES, Kathryn. Analysis of Asynchronous Online Discussion using the SOLO Taxonomy. Australian Journal of Educational \& Developmental Psychology, Newcastle, v. 5, p. 117-127, 2005.

KENNEDY, Cathleen. Models and Tools for Drawing Inferences from Student Work. Berkeley: Center University of California; Berkeley Evaluation \& Assessment Research (BEAR). Paper presented at the Annual Meeting of the American Education Research Association. Montreal, Canada, April, 2005.

MAGUIRE, T. O. The use of the SOLO taxonomy for evaluating a program for gifted students. Armidale, NSW, dez, 1988.

MEAGHER-LUNDBERG, P.; BROWN, G. T. L. Item signature study: Report on the characteristics of reading texts and items from calibration 1. Auckland, NZ: University of Auckland, Project asTTle. Technical Report, n.12, 2001.

McMILLAN, J. H. Classroom assessment. principles and practice for effective instruction. 2. ed. Boston, MA: Allyn \& Bacon, 2001.

MORSE, J. et al.Verification strategies for establishing reliability and validity in qualitative research. International Journal of Qualitative Methods, Canadá, v.1, 1 Issue 2, p. 1-19, Spring, 2002.

PANIZZON, Debra. Using a cognitive structural model to provide new insights into students' understandings of diffusion. International Journal of Science Education, Amsterdam, v. 25, n. 12, p. 1427-1450, 2003. RAPPOLT-SCHLICHTMANN, Gabrielle; et al. Transient and Robust Knowledge: contextual support and the dynamics of children's reasoning about density. Mind, Brain and Education, Cambridge, v. 1, n. 2, June, p. 98-108. 2007.

SAGLAM-ARSLAN, Aysegul. Cross-Grade Comparison of Students' Understanding of Energy Concepts. Journal of Science Educational and Technology, [Netherlands], n. 19, p. 303 - 313, 2010.

SCHWARTZ, M. S.; FISCHER, K. W. Building general knowledge and skill: cognition and microdevelopment in schince learning. In: DEMETRIOU, A.; RAFTOPOULOS, A. (Eds.). Cognitive developmental change: theories, models, and measurement. Cambridge (U.K.): Cambridge University Press, 2004.

diSESSA, Andrea; LEVRINI, Olivia. How students learn form multiple contexts and definitions: Proper time as a coordination class. Physical Review Special Topics- Physics Education Research, v.4, n.1, p. 1-10, 2008.

THOMAS, G., Holton, D., TAGG, A.; BROWN, G. T. L. Numeracy item signature study: A theoretically derived basis. Auckland, NZ: University of Auckland, Project asTTTle. Technical Report, n. 25, 2002.

Data de Recebimento: 01/02/2011

Data de Aprovação: 08/09/2011

Data da Versão Final: 16/10/2011 